\title{
A nonconjugated radical polymer with stable red luminescence in solid state
}

Zhaoyu Wang ${ }^{1,8}$, Xinhui Zou ${ }^{1,8}$, Yi Xie ${ }^{2,8}$, Haoke Zhang ${ }^{1}$, Lianrui Hu${ }^{1}$, Christopher C. S. Chan ${ }^{1}$, Ruoyao Zhang ${ }^{1}$, Jing Guo ${ }^{3}$, Ryan T. K. Kwok ${ }^{1}$, Jacky W. Y. Lam ${ }^{1}$, Ian D. Williams ${ }^{1}$, Zebing Zeng $^{3}$, Kam Sing Wong ${ }^{1}$, C. David Sherrill ${ }^{2}$, Ruquan Ye ${ }^{4 *}$, and Ben Zhong Tang ${ }^{1,5,6,7 *}$

1. Department of Chemistry, Hong Kong Branch of Chinese National Engineering Research Center for Tissue Restoration and Reconstruction and Institute for Advanced Study, and Department of Chemical and Biological Engineering, and Department of Physics, The Hong Kong University of Science and Technology (HKUST), Clear Water Bay, Kowloon, Hong Kong, China.

2. Center for Computational Molecular Science and Technology, School of Chemistry and Biochemistry, Georgia Institute of Technology, Atlanta, Georgia 30332-0400, USA.

3. State Key Laboratory of Chemo/Biosensing and Chemometrics, College of Chemistry and Chemical Engineering, Hunan University, Changsha 410082, P. R. China.

4. Department of Chemistry, City University of Hong Kong, Hong Kong, China.

5. HKUST-Shenzhen Research Institute, No. 9 Yuexing 1st RD, Nanshan District, Shenzhen 518057, China.

6. Center for Aggregation-Induced Emission, State Key Laboratory of Luminescent Materials and Devices, SCUT-HKUST Joint Research Institute, South China University of Technology, Tianhe District, Guangzhou 510640, China.

7. AIE Institute, Guangzhou Development District, Huangpu, Guangzhou 510530, China

8. These authors contributed equally to this work: Zhaoyu Wang, Xinhui Zou, Yi Xie *email: ruquanye@cityu.edu.hk; tangbenz@ust.hk

Luminescent organic radicals have attracted much attention due to its distinctive openshell structure and all-in-one properties on optoelectronics ${ }^{1}$, electronics ${ }^{2}$, and magnetics ${ }^{3}$. However, organic radicals are usually instable ${ }^{4}$ and only very limited stable structures with $\pi$-radicals can exhibit luminescent property in the isolated state, most of which originate from the family of triphenylmethyl derivatives ${ }^{5-7}$. Here, we report an unusual radical luminescence phenomenon that nonconjugated radical polymer can readily emits red luminescence at $\sim 635 \mathrm{~nm}$ in the solid state. A traditional luminescence quencher, 


\section{2,2,6,6-tetramethylpiperidine 1-oxyl (TEMPO) ${ }^{8}$, was turned into a red chromophore}

when grafted onto a polymer backbone. Experimental data confirms the emission is associated with the nitroxide radicals and is also affected by the packing of polymer. As a proof of concept, a biomedical application in intracellular ascorbic acid visualization is demonstrated. This work discloses a novel class of luminescent radicals and provides a distinctive and simple pathway for stable radical luminescence.

\section{Introduction}

Synthetic organic chromophores are commonly featured with extended $\pi$-conjugation and closed-shell structure, but their synthesis could be tedious and challenging 9 . Most of the radicals with open-shell structure are not stable, and diligent efforts have been made to stabilize the unpaired electron by delicate structure design ${ }^{10,11}$. Yet they typically relax via a nonradiative decay pathway upon excitation, thereby showing non-luminescent property ${ }^{5}$. Since the first reported case in $2006^{12}$, luminescent radicals have been widely investigated from excited-states dynamics/mechanisms ${ }^{13,14}$ to applications ${ }^{15-17}$. Nevertheless, luminescence from stable radicals remains a sporadic phenomenon and most of the structures are limited to triphenyl methyl radical derivatives and their analogues ${ }^{7,18-21}$.

In nature, non-covalent interaction and self-assembly are playing a critical role in photophysical properties. ${ }^{22,23}$ Modern photophysics suggests that in addition to the intrinsic energy states of chromophores, their luminescent properties could be affected or even reversed by the surrounding ${ }^{24,25}$. Here, we report that TEMPO, a non-luminescent radical ${ }^{5}$, could be 
transformed into a red-emissive radical after polymerization. The polymer is free from any conjugation and aromatic rings, yet the existence of narrow highest occupied molecular orbital to singly occupied molecular orbital (HOMO-SOMO) gap of nitroxide radical enables the emission in long wavelength. Experimental and theoretical data underscore the significance of intermolecular non-covalent interactions among TEMPO units. Our results disclose an unusual luminescence phenomenon and advances the development of luminescent radicals.

\section{Results and discussion}

The non-conjugated radical polymer, poly(4-glycidyloxy-2,2,6,6-tetramethylpiperidine-1-oxyl) (PGTEMPO), was synthesized via the ring-opening polymerization of stable radical monomers initiated by potassium tert-butoxide (Figure 1a). The TEMPO derivative, 4-glycidyloxy2,2,6,6-tetramethylpiperidine-1-oxyl (GTEMPO), was used as the monomer as it is stable at a wide range of temperatures and easy to crystalize. PGTEMPO is orange and it has a numberaverage molecular weight of $4.9 \mathrm{~kg} \mathrm{~mol}^{-1}$ and a narrow molecular weight distribution $(Ð=$ 1.32). It is readily soluble in common organic solvents, such as tetrahydrofuran (THF), chloroform, dichloromethane, and dimethylsulfoxide. Electron paramagnetic resonance (EPR) spectroscopy and attenuated total reflectance Fourier transform infrared (ATR-FTIR) spectroscopy confirm the existence of stable radical and nitroxide function group respectively (Figure S3 and Figure S4). Besides, the thermal property of PGTEMPO was characterized by thermogravimetric analysis (TGA), presenting a degradation temperature $\left(T_{\mathrm{d}}\right)$ of $\sim 246{ }^{\circ} \mathrm{C}$ 
(Figure S5).

a
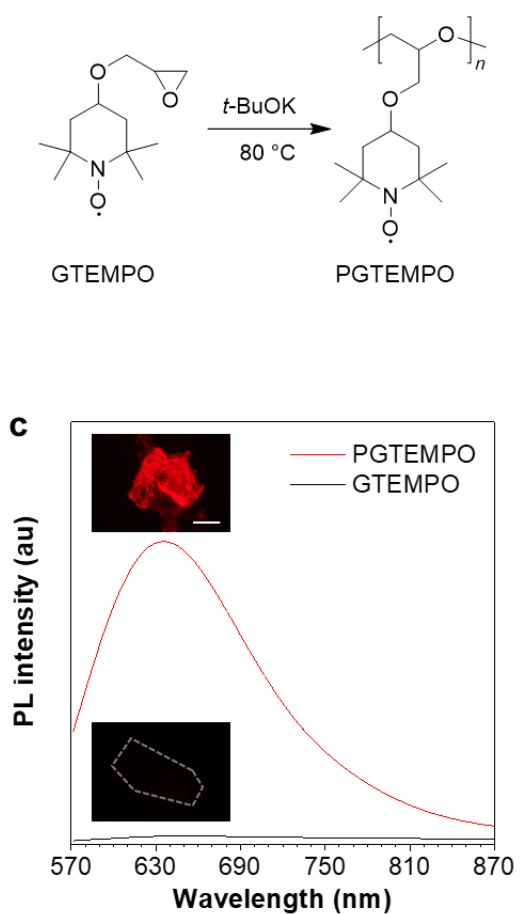
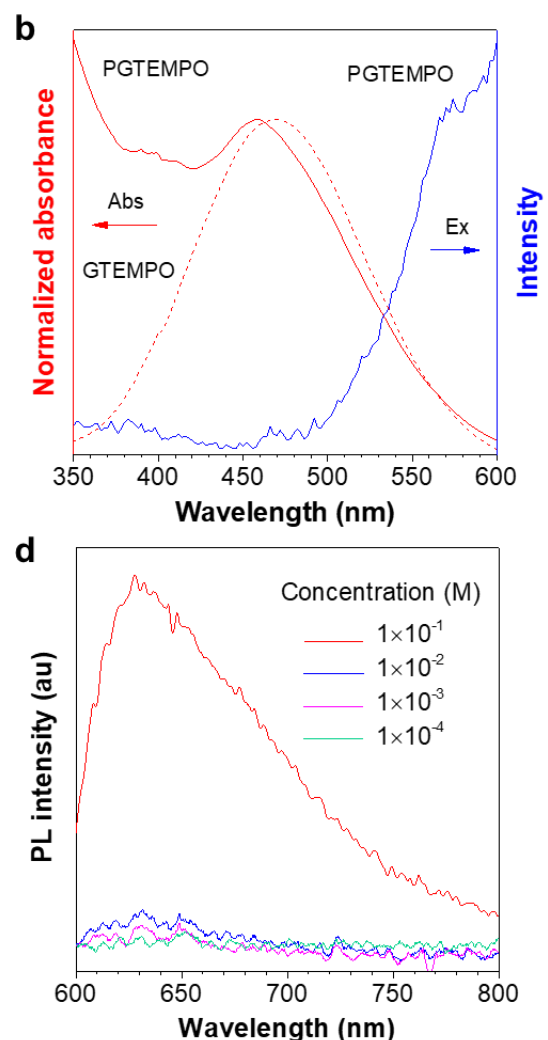

Fig. 1 Synthesis and photophysical properties. a, Synthetic route of the PGTEMPO radical polymer. b, Normalized absorption spectra of PGTEMPO (red solid line) and GTEMPO (red dash line) in THF solution; excitation spectrum of PGTEMPO solid (blue line) at emission peak of $635 \mathrm{~nm}$. c, PL spectra of PGTEMPO and GTEMPO solid excited at $532 \mathrm{~nm}$. Insets are their photos taken under 510-560 nm excitation. Scale bar: $200 \mu \mathrm{m}$. d, PL spectra of PGTEMPO in THF solution at various concentration. Excitation: $532 \mathrm{~nm}$.

Absorption spectra of PGTEMPO and GTEMPO in THF solution showed similar absorption maxima ( $\lambda_{\mathrm{abs}}$ ), which are located at about $459 \mathrm{~nm}$ and $468 \mathrm{~nm}$, respectively (Figure $1 \mathrm{~b}$ ). No PL emission signal was detected from the GTEMPO monomer. Yet for PGTEMPO, a red emission with peak intensity at $\sim 635 \mathrm{~nm}$, a quantum yield of $1.3 \%$ and a lifetime of $0.198 \mathrm{~ns}$ emerged in the solid state under the excitation of $532 \mathrm{~nm}$ (Figure 1c). The excitation spectra of PGTEMPO was obtained at the fixed emission peak of $635 \mathrm{~nm}$ as shown in Figure 1b, which does not align 
with the absorption spectra. The photographs were taken under various excitation channel (Figure S6), which further confirms that the monomer is non-luminescent under a broad range of irradiation. Then, the PL spectra of PGTEMPO were measured in THF at various concentration from $0.1 \mathrm{mM}$ to $100 \mathrm{mM}$ (Figure 1d). At low concentration, PGTEMPO displayed negligible emission. However, when the concentration increased to a threshold of $0.1 \mathrm{M}$, the emission intensity was boosted by 10 folds, demonstrating a typical aggregationinduced emission (AIE) property ${ }^{26}$. From the PL data, it is hypothesized that the emission of PGTEMPO comes from intermolecular interactions. At low concentration, the population of intermolecular interactions is low, which accounts for the faint emission. When the concentration reaches the threshold, the intermolecular interactions enhance, which turns on the luminescence. 

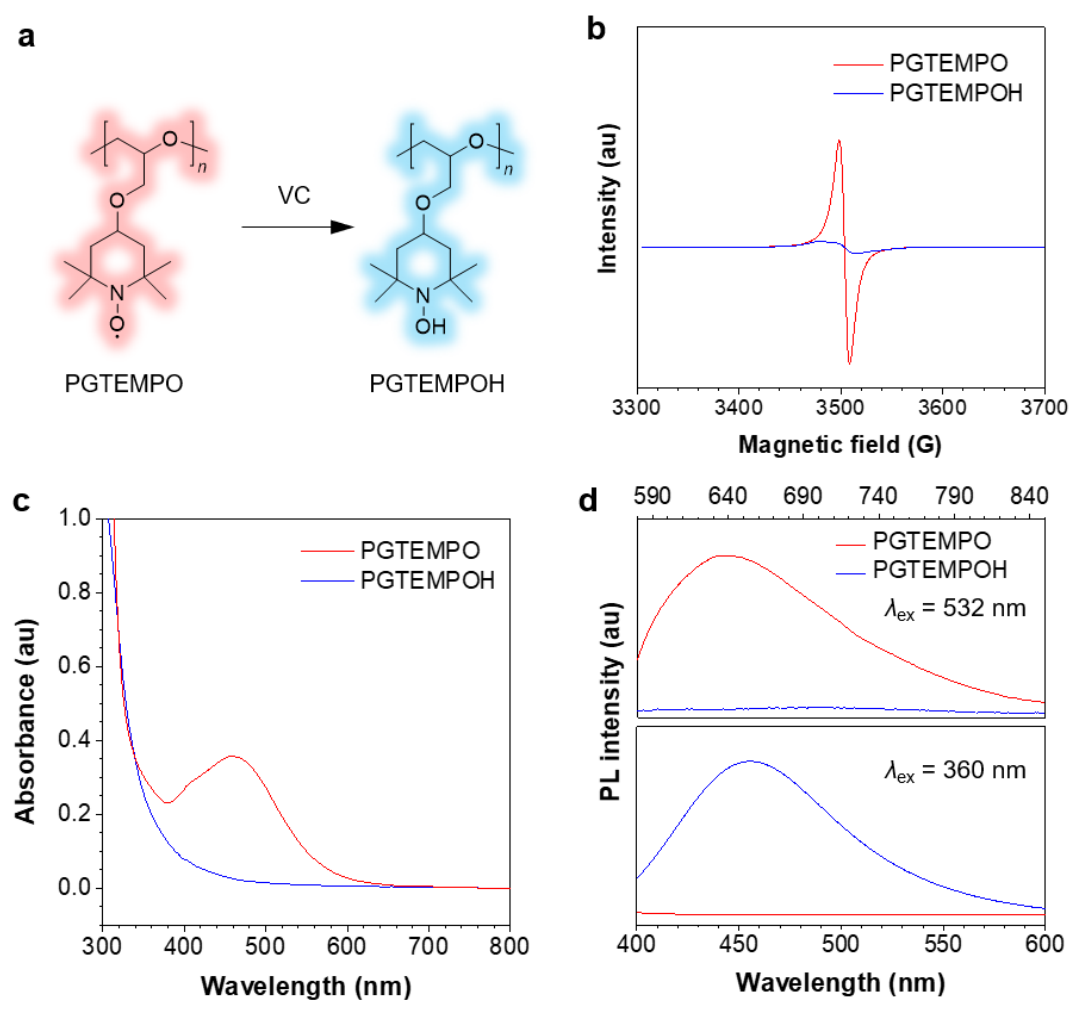

Fig. 2. The role of nitroxide radical in the photophysical property of PGTEMPO. a, Reaction scheme of PGTEMPO with VC to form PGTEMPOH. b, The EPR signal of PGTEMPO and PGTEMPOH in the solid state. c, UV-vis spectra of PGTEMPO and PGTEMPOH in DMSO (20 mM). d, PL spectra of PGTEMPO and PGTEMPOH in the solid state under an excitation wavelength of $532 \mathrm{~nm}$ and $360 \mathrm{~nm}$ respectively.

To confirmed that the luminescence is associated with the radical on TEMPO, we first designed an experiment to chemically quench the radical site on PGTEMPO with acid (vitamin C, VC) ${ }^{27}$ and observed the subsequent luminescence change. After reacting with $\mathrm{VC}$, the nitroxide group (N-O) was reduced into hydroxylamine group (N-OH), generating poly(4-glycidyloxy-2,2,6,6tetramethylpiperidine-1-hydroxyl) (PGTEMPOH) (Figure 2a). The suppression of EPR signal (Figure $2 b$ ) and the emergence of NMR spectrum of PGTEMPOH (Figure S9) suggested the successful quenching of radicals. The orange color of PGTEMPO solution also faded to colorless after reduction into PGTEMPOH (Figure 2c). As we expected, the red-emissive peak 
of PGTEMPO was significantly weakened along with the quenching of radicals (Figure 2d, top). The response to $\mathrm{VC}$ is also very sensitive and rapid (Figure S10). On the other hand, the quenching of radical generates PGTEMPOH, which is a classic clusteroluminogen ${ }^{28}$. Previous study suggests that the clustering effect from the inter/intramolecular hydrogen bond interactions could trigger the emission. ${ }^{29}$ As expected, we observed a blue emission from PGTEMPOH under excitation of $360 \mathrm{~nm}$, which is absent from PGTEMPO (Figure 2d, bottom). The luminescence quenching experiment proves that radical is playing a crucial role in the unusual red-luminescence property of PGTEMPO.

To understand the origin of luminescence from TEMPO units, we studied the dependence of luminescence on polymer packing. A cycle of temperature-dependent PL was performed between -20 and $50{ }^{\circ} \mathrm{C}$, and compared to the differential scanning calorimetry (DSC) result. The PGTEMPO presents a glass transition temperature $\left(T_{\mathrm{g}}\right)$ of $17.40{ }^{\circ} \mathrm{C}$. In general, the luminescence intensity decreases as the temperature increases, which is because of the favourable non-radiative decay at higher temperature ${ }^{30}$. Surprisingly, there is a significant drop of PL intensity between 7 to $17{ }^{\circ} \mathrm{C}$, where the polymer undergoes a glassy transition. It is hypothesized that the glassy transition breaks the rigidity of polymer, which decreases the intensity ${ }^{31}$. Besides, the real-time monitoring of the luminescence of PGTEMPO at $80^{\circ} \mathrm{C}$ under $\mathrm{N}_{2}$ was performed to probe the dynamic structural evolution of PGTEMPO. As high temperature will boost the non-radiative decay, the maximum PL intensity decreased rapidly 
within the first $20 \mathrm{~min}$. Serendipitously, the PL intensity rebounded afterwards. Previous Monte Carlo simulations suggested that the annealing will form a continuous percolation network among TEMPO units for charge transport ${ }^{32}$. Therefore, it is plausible that the increasing population of through-space interaction among the TEMPO units stimulated by the annealing process account for the escalating PL intensity. This is also supported by the observation of a red shift from 635 to $647 \mathrm{~nm}$ during the real-time annealing (Figure $3 \mathrm{c}$ ).

To further understand the luminescence mechanism of PGTEMPO, we combined the structural information and calculations. We first investigate the properties of the monomer, GTEMPO. The X-ray diffraction analysis revealed that the GTEMPO powder is orderly packed (Figure S11). The single crystal structure of GTEMPO was obtained as shown in Figure S12. In the side view, the nitroxide groups are sterically hindered by the surrounding methyl groups. The nearest distance between two nitroxide groups is $5.817 \AA$. If we term the nitroxide site as the head of the molecule, from the top view, one can observe that GTEMPO adopts a head-to-tail model, and the distance between adjacent nitroxide groups is $6.140 \AA$. Then we use timedependent density functional theory (TDDFT) calculations with a B3LYP functional and def2TZVP basis set via Q-Chem, to reveal the orbital states. The ground state of TEMPO is a doublet $\left(D_{0}\right)$ due to the existence of an unpaired electron. As depicted in Figure S13, $44 \alpha$ refers to the SOMO. The calculated $\mathrm{D}_{1}$ energy of TEMPO is $2.754 \mathrm{eV}(459 \mathrm{~nm})$, which agrees with the experimental absorption data (Figure $1 \mathrm{~b}, 468 \mathrm{~nm}$ ) and is attributed to the HOMO-SOMO 
transition.
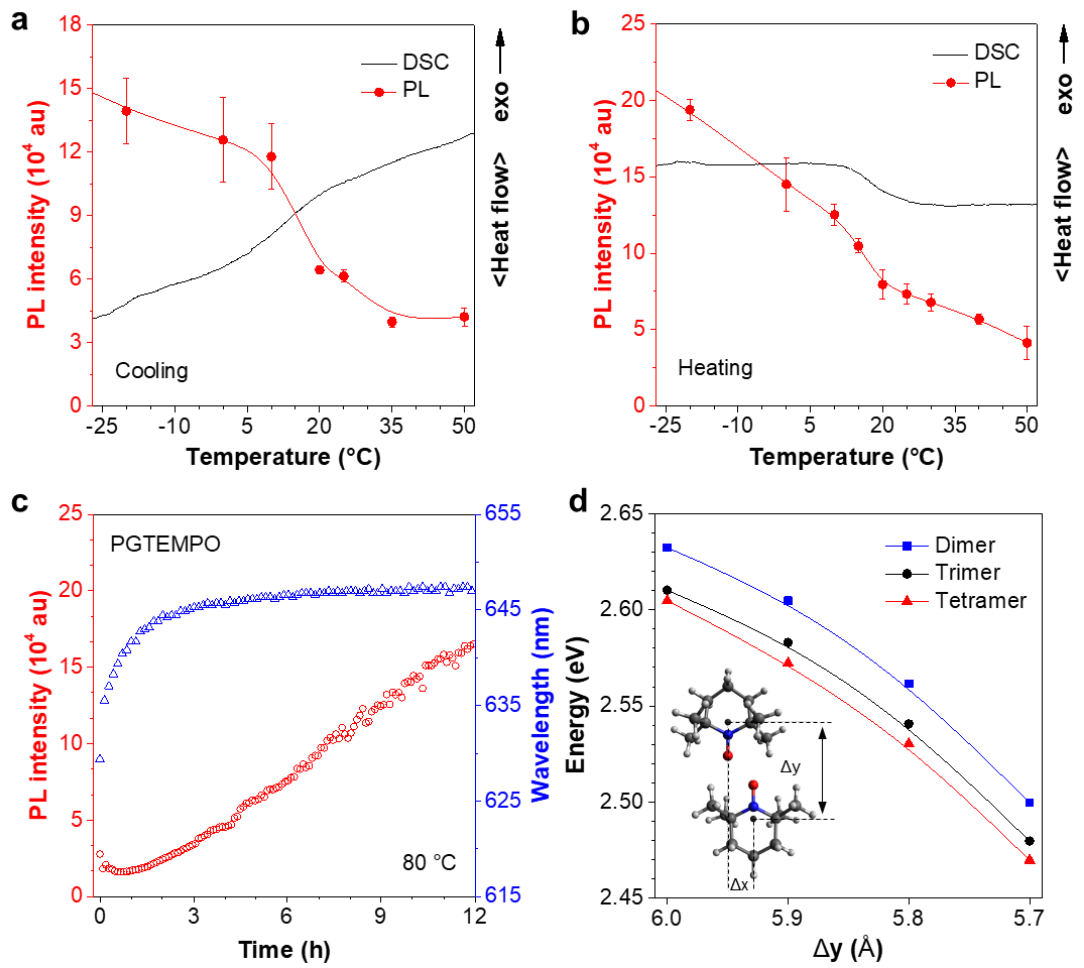

Fig. 3. | Structure-dependent photophysical properties of PGTEMPO. a-b, The PL intensity of unannealed PGTEMPO under various temperature in combination with differential scanning calorimetry thermograms recorded under nitrogen at a rate of $10{ }^{\circ} \mathrm{C} / \mathrm{min}$. c, The realtime annealing of PGTEMPO at the temperature of $80{ }^{\circ} \mathrm{C}$ under. Excitation: $532 \mathrm{~nm}$. d, First excitation energy of TEMPO cluster (dimer, trimer, and tetramer) at various separation distance.

In comparison, for PGTEMPO, the backbone of polymer readily breaks the orderly packed conformation, as shown by the powder X-ray diffraction (XRD) results that the fresh PGTEMPO sample lose the fine peaks and became completely amorphous after annealing at $80{ }^{\circ} \mathrm{C}$ (Figure S14).

To understand the energy state of PGTEMPO, we first simulate the structure by aligning the TEMPO dimer to form a parallelogram. ${ }^{32,33}$ We further added extra TEMPO units near the parallelogram to form trimer and tetramer clusters to model the effect of multi-unit clusters. 
For convenience, we defined the y-axis of the geometry as the line running through the atoms $\mathrm{N} 1$ and $\mathrm{C} 4$, and the $\mathrm{x}$-axis as the line running through atoms C3 and C5 (Figure S16a). We used the displacement between the TEMPO unit on $\mathrm{x}$-axis $(\Delta \mathrm{x})$ and $\mathrm{y}$-axis $(\Delta \mathrm{y})$ to specify the configuration of such TEMPO dimers (Figure S16b). Trimers and tetramers are constructed by placing the extra TEMPO unit above and below the nitroxide radical parallelogram plane in the dimer (Figure S16c). For all dimers in this section, we choose the value of $\Delta \mathrm{x}$ to be $1.5 \AA$ and $\Delta \mathrm{y}$ to be between $5.5 \AA$ and $6.0 \AA$, so that the two TEMPO units can approach each other to form the parallelogram between nitroxide radicals, while avoiding direct collision between the radicals and methyl groups on $\mathrm{C} 2$ and $\mathrm{C} 6$. The ground state frontier orbitals of TEMPO dimer, trimer, and tetramer were shown in Figure S17, Figure S18, and Figure S19, respectively. The excitation energy of cluster at various distance was calculated and plotted as shown in Figure $3 \mathrm{~d}$. The result shows that the energy gap decreases as the value of $\Delta \mathrm{y}$ decreases and the clustering size increases. This suggests that the existence of through-space interaction will form a new through-space cluster in the polymer with narrower HOMO-SOMO gap. It agrees with the excitation spectrum that the luminescence is induced by excitation of long wavelength (Figure 1b). In addition, it also explains the red-shift emission of annealed PGTEMPO (Figure 3c), as according to the Monte Carlo simulation $^{32}$ that annealing will favor the TEMPO clustering. 
a
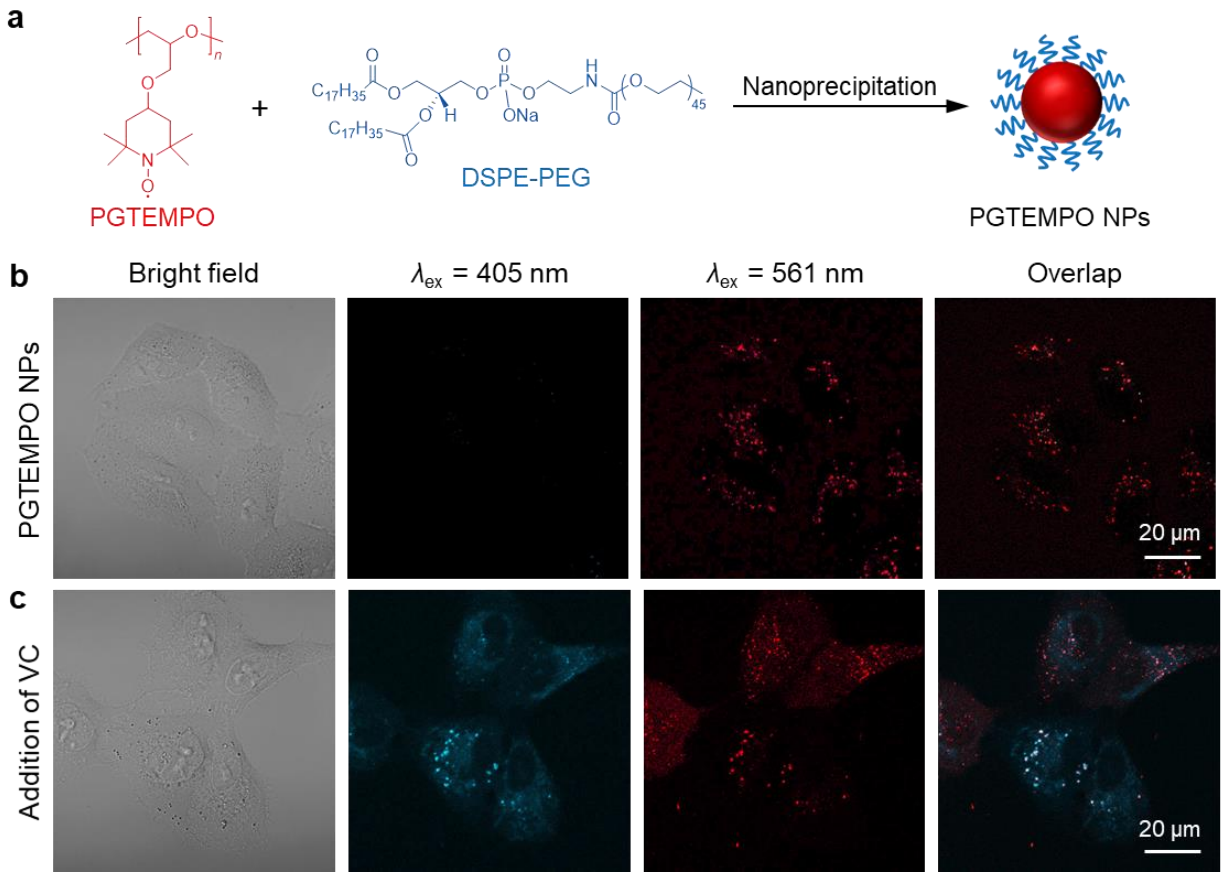

Fig. 4. | Intracellular Vitamin C detection. a, Schematic preparation of PGTEMPO NPs via a nanoprecipitation method by using an amphiphilic block copolymer DSPE-PEG as the encapsulation materials. b-c, Confocal laser scanning microscope images of A549 cell after incubation with (b) PGTEMPO NPs $(10 \mu \mathrm{g} / \mathrm{mL})$ for $4 \mathrm{~h}$ and (c) after addition with VC medium solution ( $1 \mathrm{mg} / \mathrm{mL})$ incubated for $15 \mathrm{~min}$. Excitation: $405 \mathrm{~nm}$ for second column and $561 \mathrm{~nm}$ for third column.

As a proof-of-concept demonstration, we used PGTEMPO as a potential fluorescent sensor for intracellular VC detection. To render the hydrophobic PGTEMPO with good intracellular biocompatibility and solubility in water, we encapsulated PGTEMPO with the assistance of surfactant, 1,2-distearoyl-sn-glycero-3-phosphoethanolamine-N-[methoxy-(polyethylene glycol) (DSPE-PEG) via nanoprecipitation, ${ }^{34}$ as schematically illustrated in Figure 3a. Briefly, the PGTEMPO and DSPE-PEG were dissolved in THF and added dropwise into an aqueous solution. After sonication, PGTEMPO self-assembles into nanoparticles (NPs). The size of hydrodynamic diameters of nanoparticles center around $95 \mathrm{~nm}$ as revealed by the dynamic light 
scattering (DLS) (Figure S20a).

To demonstrate the viability of VC sensing in cells, we first test the fluorescent response of PGTEMPO NPs to VC in water. The reaction of PGTEMPO NPs with VC is very fast in room temperature and aqueous solution. We obtained a peak at around $450 \mathrm{~nm}$ after the addition of VC solution to PGTEMPO NPs (Figure S20b-c). Afterwards, we explored the in vitro cellular uptake and VC mapping performance of PGTEMPO NPs using A549 lung cancer cells as an example. After incubation for $4 \mathrm{~h}$, we observed a substantial accumulation of PGTEMPO NPs inside the A549 cells via confocal laser scanning microscope. In the image of Figure $4 \mathrm{~b}$ under excitation of $561 \mathrm{~nm}$, red luminescence was observed. Subsequently, we added VC to medium and incubated the cells for $15 \mathrm{~min}$. The fluorescent signal in cells under irradiation of $405 \mathrm{~nm}$ emerged in Figure 3c, confirming that PGTEMPO NPs could serve as a turn-on probe for intracellular mapping of VC distribution. To further confirm that PGTEMPO NPs targeted at lysosome, the colocalization experiment was carried out with commercial LysoTracker Green (LTG), a lysosome marker with short excitation/emission of $488 / 511 \mathrm{~nm}$ as the control. As shown in Figure S21, the fine lysosome structures from PGTEMPO NPs greatly overlap with those from LTG, confirming the specific lysosome targeting of PGTEMPO NPs. The fluorescent signal in cells indicates that VC diffused in cells swiftly $(<15 \mathrm{~min})$ and can enter lysosomes. In comparison with reported $\mathrm{VC}$ fluorescent probe from one-channel imaging ${ }^{35}$, the dual fluorescent signals (under $561 \mathrm{~nm}$ and $405 \mathrm{~nm}$ channel) from PGTEMPO NPs improve 
the imaging reliability.

\section{Conclusions}

We have synthesized a nonconjugated radical polymer showing luminescence in the solid state.

To our knowledge, this is the first report that stable radical without any conjugated or aromatic structures can emit light. Luminescence quenching experiment confirms the key role of nitroxide radicals. Combining the structural information and calculation, we propose that the intermolecular interactions of TEMPO clusters account for the long-wavelength emission. Using the redox characteristic of TEMPO, a biological application of intracellular VC visualization was demonstrated. This work expands the family of luminescent radicals. We envision that further experimental and theoretical researches on this unconventional luminescence phenomenon will provide insights into principles governing radical luminescence and find applications in broad fields.

\section{References}

1. Ai, X. et al. Efficient radical-based light-emitting diodes with doublet emission. Nature 563, 536-540 (2018).

2. Guo, H. et al. High stability and luminescence efficiency in donor-acceptor neutral radicals not following the Aufbau principle. Nat. Mater. 18, 977-984 (2019).

3. Kimura, S. et al. Magnetoluminescence in a Photostable, Brightly Luminescent Organic Radical in a Rigid Environment. Angew. Chemie 57, 12711-12715 (2018).

4. Peng, Q., Obolda, A., Zhang, M. \& Li, F. Organic light-emitting diodes using a neutral $\pi$ radical as emitter: The emission from a doublet. Angew. Chemie - Int. Ed. 54, 70917095 (2015).

5. Teki, Y. Excited-State Dynamics of Non-Luminescent and Luminescent $\pi$-Radicals. Chem. - A Eur. J. 26, 980-996 (2020). 
6. Abdurahman, A., Peng, Q., Ablikim, O., Ai, X. \& Li, F. A radical polymer with efficient deep-red luminescence in the condensed state. Mater. Horizons 6, 1265-1270 (2019).

7. Ai, X., Chen, Y., Feng, Y. \& Li, F. A Stable Room-Temperature Luminescent Biphenylmethyl Radical. Angew. Chemie - Int. Ed. 57, 2869-2873 (2018).

8. Rivera, S. A. \& Hudson, B. S. Rapid exchange luminescence: Nitroxide quenching and implications for sensor applications. J. Am. Chem. Soc. 128, 18-19 (2006).

9. Xu, W., Wang, D. \& Tang, B. Z. NIR-II AIEgens: A Win-Win Integration towards Bioapplications. Angew. Chemie (2020).

10. Armet, O. et al. Inert carbon free radicals. 8. Polychlorotriphenylmethyl radicals. Synthesis, structure, and spin-density distribution. J. Phys. Chem. 91, 5608-5616 (1987).

11. Ballester, M., Riera, J., Castafier, J., Badía, C. \& Monsó, J. M. Inert carbon free radicals. I. Perchlorodiphenylmethyl and perchlorotriphenylmethyl radical series. $J$. Am. Chem. Soc. 93, 2215-2225 (1971).

12. Gamero, V. et al. [4-(N-Carbazolyl)-2,6-dichlorophenyl]bis(2,4,6trichlorophenyl)methyl radical an efficient red light-emitting paramagnetic molecule. Tetrahedron Lett. 47, 2305-2309 (2006).

13. Kato, K., Kimura, S., Kusamoto, T., Nishihara, H. \& Teki, Y. Luminescent RadicalExcimer: Excited-State Dynamics of Luminescent Radicals in Doped Host Crystals. Angew. Chemie - Int. Ed. 58, 2606-2611 (2019).

14. Ito, A. et al. Excited-State Dynamics of Pentacene Derivatives with Stable Radical Substituents. Angew. Chemie 126, 6833-6837 (2014).

15. Badalyan, A. \& Stahl, S. S. Cooperative electrocatalytic alcohol oxidation with electron-proton-transfer mediators. Nature 535, 406-410 (2016).

16. Shimizu, A., Ito, A. \& Teki, Y. Photostability enhancement of the pentacene derivative having two nitronyl nitroxide radical substituents. Chem. Commun. 52, 2889-2892 (2016).

17. Rajca, A. et al. Organic radical contrast agents for magnetic resonance imaging. J. Am. Chem. Soc. 134, 15724-15727 (2012).

18. Hattori, Y., Kusamoto, T. \& Nishihara, H. Enhanced luminescent properties of an open-shell (3,5-Dichloro-4-pyridyl)bis(2,4,6-trichlorophenyl)methyl radical by coordination to gold. Angew. Chemie - Int. Ed. 127, 3802-3805 (2015).

19. Dong, S. et al. Multicarbazolyl substituted TTM radicals: Red-shift of fluorescence emission with enhanced luminescence efficiency. Mater. Chem. Front. 1, 2132-2135 (2017).

20. Heckmann, A. et al. Highly fluorescent open-shell NIR dyes: The time-dependence of back electron transfer in triarylamine-perchlorotriphenylmethyl radicals. J. Phys. 
Chem. C 113, 20958-20966 (2009).

21. Velasco, D. et al. Red organic light-emitting radical adducts of carbazole and tris(2,4,6-trichlorotriphenyl)methyl radical that exhibit high thermal stability and electrochemical amphotericity. J. Org. Chem. 72, 7523-7532 (2007).

22. Shimomura, O. \& Johnson, F. H. Calcium binding, quantum yield, and emitting molecule in aequorin bioluminescence. Nature 227, 1356-1357 (1970).

23. Shimomura, O., Johnson, F. H. \& Saiga, Y. Extraction, Purification and Properties of Aequorin, a Bioluminescent Protein from the Luminous Hydromedusan, Aequorea. $J$. Cell. Comp. Physiol. 59, 223-239 (1962).

24. Zhao, Z., Zhang, H., Lam, J. W. Y. \& Tang, B. Z. Aggregation-Induced Emission: New Vistas at the Aggregate Level. Angewandte Chemie (2020).

25. Sun, P. et al. J-Aggregate squaraine nanoparticles with bright NIR-II fluorescence for imaging guided photothermal therapy. Chem. Commun. 54, 13395-13398 (2018).

26. Wang, Q. et al. Reevaluating Protein Photoluminescence: Remarkable Visible Luminescence upon Concentration and Insight into the Emission Mechanism. Angew. Chemie 58, 12667-12673 (2019).

27. Tang, Y. et al. Radical scavenging mediating reversible fluorescence quenching of an anionic conjugated polymer: Highly sensitive probe for antioxidants. Chem. Mater. 18, 3605-3610 (2006).

28. Zhang, H. et al. Clusterization-triggered emission: Uncommon luminescence from common materials. Mater. Today 32, 275-292 (2020).

29. Ye, R. et al. Non-conventional fluorescent biogenic and synthetic polymers without aromatic rings. Polym. Chem. 8, 1722-1727 (2017).

30. Cui, Y., Zhu, F., Chen, B. \& Qian, G. Metal-organic frameworks for luminescence thermometry. Chem. Commun. 51, 7420-7431 (2015).

31. Leung, N. L. C. et al. Restriction of intramolecular motions: The general mechanism behind aggregation-induced emission. Chem. - A Eur. J. 47, 15349-15353 (2014).

32. Joo, Y. et al. A nonconjugated radical polymer glass with high electrical conductivity. Science 359, 1391-1395 (2018).

33. Zhang, H. et al. In situ monitoring of molecular aggregation using circular dichroism. Nat. Commun. 9, 4961 (2018).

34. Cai, X. et al. Multifunctional Liposome: A Bright AIEgen-Lipid Conjugate with Strong Photosensitization. Angew. Chemie 130, 16634-16638 (2018).

35. Ishii, K., Kubo, K., Sakurada, T., Komori, K. \& Sakai, Y. Phthalocyanine-based fluorescence probes for detecting ascorbic acid: Phthalocyaninatosilicon covalently linked to TEMPO radicals. Chem. Commun. 47, 4932-4934 (2011). 


\section{Methods}

\section{Synthesis of GTEMPO}

The monomer, GTEMPO, was synthesized as reported ${ }^{36}$. Briefly, TEMPO-OH was purified by recrystallization before use. Sodium hydroxide $(\mathrm{NaOH})(8 \mathrm{~g})$ was gradually added to deionized water $(16 \mathrm{~mL})$ in a $250 \mathrm{~mL}$ round-bottom flask under vigorous stirring. After $\mathrm{NaOH}$ was completely dissolved, epichlorohydrin $(10 \mathrm{~mL}, 120 \mathrm{mmol})$ and TBA $(1.5 \mathrm{~g}, 4.6 \mathrm{mmol})$ were added. A solution of TEMPO-OH (4.12 g, $24 \mathrm{mmol}$ ) in $20 \mathrm{~mL}$ tetrahydrofuran (THF) was then added dropwise into the mixture. The resulting solution was stirred at room temperature overnight. The reaction mixture was poured into $200 \mathrm{~mL}$ of ice water and then extracted with ethyl acetate (EA). The organic layer was washed with sodium chloride $(\mathrm{NaCl})$ aqueous solution and then extracted with ethyl acetate again. The combined organic layers were then dried over anhydrous sodium sulfate. After filtration, the filtrate was evaporated under reduced pressure and the crude product was purified on a silica gel column using hexane/EA (8/1, v/v) as the eluent. The oily product obtained was freeze-dried for 1 day to yield the monomer, GTEMPO, as a red crystalline solid.

\section{Synthesis of PGTEMPO}

The polymerization of the monomer was achieved using a procedure optimized from the literature $^{37}$. GTEMPO was further dried under reduced pressure for one day before use and stored in glove box. Inside a glove box, a mixture of GTEMPO (300 $\mathrm{mg}, 1.31 \mathrm{mmol}$ ) and potassium tert-butoxide (12 mg) were added into a $10 \mathrm{~mL}$ Schlenk tube with a stirring bar, which was already dried in a hot oven overnight. Then, the tube was sealed with a rubber stopper. The reaction mixture was heated at $80{ }^{\circ} \mathrm{C}$ for 2 hours without solvent and then was injected with $2 \mathrm{~mL}$ anhydrous THF for another 8 hours. The mixture was vortexed after addition of solvent to make sure the mixture was well dissolved in THF. After cooling down to room temperature, $\mathrm{NaCl}$ aqueous solution was added to mixture, followed by extraction with chloroform for three times. The organic solvent was removed under reduced pressure and the crude polymer dissolved with a small volume of THF. The crude polymer solution was passed through a simple column filled with neutral $\mathrm{Al}_{2} \mathrm{O}_{3}$ powder and precipitated in hexane. The precipitates were collected by ultracentrifugation (7000 rpm for $3 \mathrm{~min}$ ). This protocol was repeated for three times to remove excess unreacted monomer. The polymer was dried overnight in a vacuum oven at room temperature to obtain an orange solid. $M_{n}=4,900 ; M_{w}=$ 6,$500 ; M_{w} / M_{n}=1.32$ (GPC, polystyrene calibration).

\section{Data Availability}

All experimental data are available in the main text or the supplementary materials. 


\section{Methods references}

36. Chang, C. et al. Synthesizing and characterization of comb-shaped carbazole containing copolymer via combination of ring opening polymerization and nitroxide-mediated polymerization. Polymer. 51, 1947-1953 (2010).

37. Endo, T. et al. Synthesis and polymerization of 4-(glycidyloxy)-2, 2, 6, 6tetramethylpiperidine-1-oxyl. Macromolecules 26, 3227-3229 (1993).

\section{Acknowledgments}

The authors are grateful for financial support from the National Science Foundation of China (21788102), the Research Grants Council of Hong Kong (16308016, C6009-17G, and AHKUST 605/16), the University Grants Committee of Hong Kong (AoE/P-03/08 and AoE/P02/12), the Innovation and Technology Commission (ITC-CNERC14SC01 and ITS/254/17), and the Science and Technology Plan of Shenzhen (JCYJ20160229205601482 and JCY20170818113602462). We would also like to thank Dr. Shunjie Liu, Dr. Qingqing Gao, Zaiyu Wang for their kind assistance. Besides, we are grateful for Dr. Herman H. Y. Sung who conducted single crystal $\mathrm{X}$-ray diffraction in this work.

\section{Author contributions}

Z. W., R. Y., and B. Z. T. conceived the idea. Z. W. synthesized the materials and completed the characterization. X. Z., Z. W., C. C. S. C., and K. S. W. performed the photophysical experiments. Y. X., L. H., and D. S. carried out the theoretical calculations and results analyses. R. Z. and Z. W. obtained the biological application experiments. J. G. and Z. Z. conducted the EPR measurement. I. W. carried out the single crystal X-ray diffraction. H. Z., R. Y. and B. Z. T. initiated and supervised the work. Z. W., R. Y., and B. Z. T. wrote the manuscript. H. Z., R. T. K. K., J. W. Y. L., and K. S. W. revised the manuscript with input from all authors.

\section{Conflicts of interests}

The authors declare no competing interests. 